\title{
RIESGOS PSICOSOCIALES EN EL LUGAR DE TRABAJO: APROXIMACIÓN TEÓRICA Y MARCO LEGAL EN URUGUAY
}

\section{PSYCHOSOCIAL RISK IN THE WORKPLACE: THEORETICAL APPROACH AND LEGAL FRAMEWORK IN URUGUAY}

\author{
David González-Trijueque \\ Tribunal Superior de Justicia de Madrid, España \\ Silvana Giachero \\ Asociación contra el Acoso Moral Laboral en Uruguay, Uruguay
}

Sabino Delgado

Plataforma contra los Riesgos Psicosociales y la Discriminación Laboral de la Comunidad de Madrid, España

\begin{abstract}
Resumen: Esta comunicación procura realizar una aproximación al concepto de riesgos psicosociales en el lugar de trabajo y abordar algunos de los supuestos más habituales, con los que puede encontrarse el psicólogo en su práctica profesional. De hecho, el principal objetivo es facilitar una introducción al tema de riesgos psicosociales en el entorno laboral y plantear algunos de esos riesgos (ej. estrés laboral, Burnout, mobbing) que puedan ser de interés para los profesionales en Psicología y Recursos Humanos. Asimismo, se describe -someramente- el contexto legal existente en Uruguay para abordar estos fenómenos. La importancia del estudio de estos factores radica en que la vigilancia y control sobre la salud laboral de los trabajadores, ha puesto de manifiesto que los riesgos presentes en el entorno laboral -y que suponen un importante peligro para la salud de las personas- no proceden exclusivamente de los factores físicos, sino que los factores psicosociales son muy importantes e influyen en la salud y el bienestar, es decir, en los estados de estrés que originan procesos físicos y / o psicológicos.

Por último, se discute sobre la importancia de la prevención de riesgos laborales, como una herramienta útil para hacer frente a este tipo de situaciones.
\end{abstract}

Palabras clave: riesgos psicosociales, estrés laboral, burnout, mobbing.

Abstract:In this paper psychosocial risks in the workplace are discussed from a theoretical point of view. In addition, the most frequent issues a psychologist faces in this context are analyzed. This article is aimed at conceptualizing the psychosocial risks in the work context and identifying the most frequent of those risks that might be of relevance for psychologists (e.g. job stress, burnout, mobbing). Additionally, the current legal framework related to these risks in Uruguay is discussed.

The relevance of the subject is evidenced by the fact that the risks faced by the workers in their workplace are not just physical in nature. Psychosocial risks are highly important and influence the workers' health and well being, particularly since stressful conditions can result in physiological and/or psychological conditions. Finally, the importance of psychosocial risks prevention is discussed.

Keywords: psychosocial risk, job stress, burnout, mobbing.

\section{INTRODUCCIÓN}

En la actualidad, un porcentaje significativo de las demandas profesionales que reciben los psicólogos, independientemente del área a la que se dediquen, son aquellas relacionadas con los factores de tipo psicosocial propios del lugar de trabajo; los que originan en los trabajadores que los padecen numerosos problemas en su salud, tanto a nivel físico como psíquico. No debemos olvidar que en toda organización de trabajo existen distintos agentes que pueden 
afectar la esfera social y psicológica de los trabajadores (Almodóvar et al., 2003), son los que se ha dado en llamar riesgos psicosociales y refieren a aspectos de la concepción, organización y gestión del trabajo, así como de su contexto social y ambiental, que tienen la potencialidad de causar daños físicos, sociales o psicológicos en los trabajadores y que en los últimos años se están convirtiendo en uno de los principales problemas de salud laboral (Llaneza, 2009). Estos riesgos psicosociales forman parte de los denominados "nuevos riesgos emergentes" y tienen su origen en unas condiciones de trabajo deficientes, principalmente de tipo organizativo. De este modo, podemos definir de manera genérica a estos riesgos como, aquellas condiciones presentes en el ámbito laboral, directamente relacionadas con la organización del trabajo, el contenido del trabajo y la realización de la tarea y que se presentan con la capacidad para afectar el desarrollo del trabajo y la salud del trabajador. De este modo, se conceptúa que los factores psicosociales son aquellas características del trabajo -y sobre todo de su organización- que afectan a la salud de las personas, a través de distintos mecanismos psicológicos o fisiológicos.

Actualmente disponemos de numerosas evidencias científicas que confirman que, la exposición a los factores de riesgo psicosocial afecta de manera significativa a la salud de los trabajadores (Almodóvar et al., 2003; Llaneza, 2005, 2009). A corto plazo, estos riesgos se manifiestan a través del concepto general de estrés laboral, que incluye diversos aspectos de la salud física, mental y social; mientras que a largo plazo, la exposición a los riesgos psicosociales puede provocar alteraciones cardiovasculares, respiratorias, inmunitarias, gastrointestinales, dermatológicas, endocrinas, musculo-esqueléticas y de la salud mental (Almodóvar et al., 2003).

Debido al carácter nocivo de estos riesgos, se antoja fundamental el velar por la salud de los trabajadores. Para ello, se debe reconocer en primer lugar el derecho de los trabajadores a la protección de su salud y de su integridad (Vida, 2003). Sin embargo, las estadísticas demuestran que este derecho está lejos de estar garantizado; las cifras de accidentes de trabajo, las bajas por enfermedad y las incapacidades laborales, indican que existen situaciones y condiciones de trabajo, en donde la seguridad en el trabajo y promoción de la salud son más una aspiración que una realidad. Unas determinadas condiciones de trabajo serán adecuadas si promueven la salud física, psíquica y social de los trabajadores (Almodóvar et al., 2003); es por ello que, en el lugar de trabajo, es el empresario (o la administración pública) quien debe tener la responsabilidad de garantizar la seguridad y la salud de los trabajadores en todos los aspectos relacionados con la actividad, siendo la evaluación de riesgos la técnica que permitirá tomar las medidas adecuadas para garantizar la salud de esos recursos humanos. Si la evaluación de riesgos pone de manifiesto que unas determinadas condiciones de trabajo deterioran la salud del trabajador, será necesario modificar esa situación a través de un programa de mejora, controlado y revisado en el tiempo (Llaneza, 2009).

\section{LA EVALUACIÓN DE LOS RIESGOS LABORALES}

En tanto se enfatiza la importancia de una evaluación de riesgos laborales, se hizo necesario enmarcar las acciones profesionales dentro de la disciplina conocida como Psicosociología Relacional; la que comprende tres momentos distintos de actuación: 1) la evaluación inicial de riesgos, 2) la evaluación periódica de riesgos y 3 ) la intervención en crisis o momentos puntuales. Evidentemente, todos estos momentos de intervención son susceptibles de ser aplicados a los distintos riesgos psicosociales.

La evaluación de los riesgos de origen psicosocial, como cualquier evaluación general de riesgos, es un proceso complejo que conlleva una serie de actuaciones o etapas sucesivas e interrelacionadas (Llaneza, 2009). En general, se coincide en que, para realizar una evaluación de factores psicosociales en el lugar de trabajo, se deben seguir las siguientes fases: 1) determinar los riesgos que se han de analizar, 2) identificar a los trabajadores expuestos a dichos riesgos, 3) seleccionar la metodología y las técnicas más apropiadas, 4) formular hipótesis de trabajo, 5) planificar y realizar el trabajo de campo, 6) analizar los resultados, 7) elaborar informes con los resultados obtenidos, 8) desarrollar y poner en marcha un programa de intervención y 9) efectuar el seguimiento y control del programa instaurado. 
Es de especial relevancia evaluar aspectos tales como, la carga mental, el grado de autonomía, el contenido del trabajo, la definición de rol o las relaciones interpersonales; esto último en razón de que, todo riesgo psicosocial se configura a partir de la interacción producida entre determinadas características propias del trabajador (ej., personalidad, base biológica, formación, aptitudes, apoyo social) y otras características que son propias de la tarea a realizar (ej., exigencia cognitiva, cantidad, clientes/usuarios, compañeros/superiores). Así, por ejemplo, cuando las características de la tarea superan los recursos del trabajador, aparece el malestar y el estrés, cuya gravedad estará -a su vez- determinada por otras variables externas (ej. tiempo de exposición, intensidad) e internas (ej. vulnerabilidad, resistencia).

Con independencia de los métodos que evalúan factores concretos y riesgos específicos de tipo psicosocial , por ejemplo el Maslach Burnout Inventory, (Maslach \& Jackson, 1981) para evaluar el Burnout o el LIPT-60 -Modificado- para la Valoración de las estrategias de acoso psicológico en el trabajo (Leymann \& Tallgren, 1989, en González de Rivera \& Rodríguez Abuín, 2005), existen una serie de técnicas generales para evaluar los factores de riesgo psicosocial. Entre estos métodos se encuentran las listas de chequeo (check-list), los cuestionarios y escalas (ej. sintomatología clínica, problemas de salud), los inventarios sobre las características personales (ej. personalidad), los indicadores bioquímicos y los indicadores electrofisiológicos.

Los distintos métodos de evaluación en esta área pueden agruparse en dos categorías: 1) los métodos cuantitativos, que consisten en cuestionarios y encuestas y que son los más utilizados y 2) los métodos cualitativos, que consisten en entrevistas y grupos de discusión y aportan gran riqueza a la información obtenida a través de los métodos cuantitativos. Éstos últimos destacan por ser los de mayor fiabilidad y validez probada y estar elaborados por entidades de acreditado y reconocido prestigio (Moncada et al., 2002). Además, son numerosos y variados en cada país, por lo que aquí únicamente se hace referencia a dos instrumentos ampliamente utilizados a nivel internacional :
- El método CoPsoQ desarrollado por el Instituto Nacional de Salud Laboral de Dinamarca y que valora distintos aspectos de interés psicosocial (Moncada, Llorens \& Kristensen, 2005).

- El método LEST, desarrollado por el Laboratorio de Economía y Sociología del Trabajo de Francia y que evalúa concretamente la carga mental a la que está expuesto el trabajador (Guélaud, Beauchesne, Gautrat \& Roustang, 1978, en Castillo et al, 1999, Llaneza, 2009).

Pese a su importancia, el manejo de los riesgos laborales de origen psicosocial se puede considerar, todavía, como una asignatura pendiente de la Prevención de los Riesgos Laborales, sin embargo, cada día se constata de forma creciente la importancia que éstos tienen sobre la salud de los trabajadores y de las organizaciones. Es por tanto, que la evaluación de riesgos psicosociales se convierte en una herramienta preventiva de gran importancia, puesto que la identificación, valoración y control de los factores de riesgo de origen psicosocial y la planificación de las medidas preventivas correspondientes, conducirá a una gestión eficaz de las personas de la organización, en el sentido de una mejor adecuación a la tarea a desempeñar, al entorno, a la empresa, y se traducirá en eficacia de la organización: mejor rendimiento, menor absentismo, mayor satisfacción, etc.

\section{CONSECUENCIAS ASOCIADAS A LOS RIESGOS PSICOSOCIALES EN EL LUGAR DE TRABAJO}

A continuación se abordan tres de las más habituales y graves manifestaciones que pueden surgir como consecuencia de los riesgos psicosociales en el lugar de trabajo y que suponen motivo de consulta habitual para los profesionales de la psicología.

\subsection{Estrés laboral}

La exposición a los factores de riesgo psicosocial tiene habitualmente como resultado la experiencia negativa del estrés laboral (Velázquez, 2005). Desde una perspectiva integradora, el estrés podría definirse como la respuesta fisiológica, psicológica y de comportamiento de un individuo que intenta 
adaptarse y ajustarse a presiones internas y externas (Del Hoyo, 2001); de hecho, el término estrés ha sido adoptado por la psicología y la medicina a partir del vocablo inglés "stress" que designa una fuerza que deforma cuerpos o provoca tensión, tratándose del proceso de adaptación a cualquier tipo de cambio que el organismo lleva a término habitualmente para recuperar su estabilidad interna (homeostasis) en todo tipo de circunstancias, es decir, se trata de un mecanismo natural de adaptación del ser humano (Del Hoyo, 2001).

Considerando el estrés como la respuesta fisiológica, psicológica y de comportamiento de un individuo que intenta adaptarse a presiones internas y externas, se debe señalar que el estrés laboral surge cuando se da un desajuste entre la persona, el puesto de trabajo y la propia organización; por ello, la persona percibe que no dispone de recursos suficientes para afrontar la problemática laboral y aparece la experiencia del estrés (Del Hoyo, 2001), tratándose por tanto de un estado psicológico que es parte y reflejo de un proceso de interacción entre la persona y su entorno laboral (Buendía \& Ramos, 2001). La exposición prolongada a situaciones estresantes en el ámbito laboral y la desadaptación que éstas producen en el organismo van a tener como consecuencia más importante la aparición de determinadas alteraciones, si bien debemos señalar que la sintomatología asociada al estrés es muy variada (ej., temblores, tensión muscular, cefaleas, sudoración, sequedad bucal, dolor abdominal, mareos, arritmias, cansancio, irritabilidad, dificultades para dormir, etc.) y estará determinada tanto por las características del estresor en cuestión como por variables moduladoras y de resistencia/vulnerabilidad del trabajador (Almodóvar et al., 2003).

En cuanto a su etiología, el estrés laboral puede ser desencadenado por diversos factores, entre los que destacan el ambiente físico de trabajo (ej., ruido, vibraciones, iluminación y temperatura), los contenidos del puesto (ej., la variedad de las tareas y la complejidad del trabajo), la definición de rol del trabajador, las relaciones interpersonales, las posibilidades de desarrollo de una carrera profesional, los aspectos organizacionales y los aspectos relacionados con las nuevas tecnologías (Buendía \& Ramos, 2001; Peiró, 1999). Además, entre las causas generadoras de estrés laboral no sólo destacan las propias del entorno objetivo del trabajador (p. ej., estresores del ambiente físico, estresores individuales, estresores grupales, estresores organizacionales y estresores extra-organizacionales), sino también las asociadas a la subjetividad del mismo (Morán, 2006); de hecho, las personas poseen unas características individuales y sociales que les hacen responder de distinta forma a las exigencias que les demanda su trabajo (Vida, 2003).

Entre las características del trabajo a desarrollar son de especial importancia en relación a la respuesta de estrés la sobrecarga cualitativa (excesivas demandas intelectuales o mentales en relación con los conocimientos y habilidades del trabajador), la sobrecarga cualitativa (la dificultad excesiva de la tarea), la subcarga cualitativa (realizar tareas muy sencillas que debido a la falta de estimulación, aburrimiento y monotonía también puede producir estrés), el escaso control sobre la tarea (grado en que un ambiente laboral permite al individuo controlar las actividades a realizar y viene dado por el grado de autonomía, iniciativa y responsabilidad) y el grado de autonomía (la mayor o menor dependencia jerárquica en el desempeño de las funciones ejecutadas) (Almodóvar et al., 2003; Llaneza, 2009). También tienen importancia los estresores relativos a la organización de trabajo, entre los que destacan la ambigüedad de rol (falta de claridad sobre el trabajo que se está desempeñando, los objetivos de ese trabajo y el alcance de las responsabilidades), los problemas de comunicación (es necesario conocer qué aspectos pueden dificultar la comunicación o impedirla), las relaciones interpersonales en el trabajo (pueden convertirse en un factor de estrés), la promoción y desarrollo de la carrera profesional (el desequilibrio entre las aspiraciones del individuo sobre su carrera profesional y el nivel real de sus logros se puede convertir en fuente de preocupación, ansiedad y frustración), la formación (concebida como los conocimientos básicos necesarios para poder cumplir la prestación laboral pactada, la formación continua recibida, la experiencia obtenida) y la participación de los trabajadores (la no participación en la toma de decisiones constituye un conjunto de elementos que influyen en gran medida en el bienestar de los trabajadores) (Almodóvar et al., 2003; Llaneza, 2009). 
Por otra parte, también se deben considerar los factores moduladores que pueden interferir ante la exposición del estrés laboral potenciando o disminuyendo sus efectos ya que el estrés se origina a partir estímulos, físicos y sociales, que someten a la persona a demandas que no puede satisfacer de forma competente y adecuada, al tiempo que percibe la necesidad de hacerlo. No obstante, las diferencias individuales juegan un papel en cuanto a la vulnerabilidad de la persona en referencia a los factores de estrés (González-Trijueque y Graña, 2010; Morán, 2006), existiendo una serie de factores personales e interpersonales susceptibles de aumentar el riesgo de estrés en el lugar de trabajo y que no sólo dependen de los rasgos propios del carácter y personalidad de cada trabajador sino de la situación concreta en cada caso. Así, dependiendo de la situación personal que se viva, el trabajador estará en disposición o no de combatir el estrés.

Los aspectos que más influyen en la percepción del estrés, son en primer lugar su propia personalidad, después influye la formación, las aspiraciones, las expectativas, la salud, la presión familiar, social, etc., y la situación económica (Almodóvar et al., 2003). Estas diferencias individuales juegan un papel importante en la experiencia del estrés laboral, ya que la combinación de una situación particular y de un individuo determinado (con sus características personales específicas, expectativas, experiencias pasadas, actitudes, aptitudes y sus propios sentimientos) puede dar como resultado una falta de equilibrio que induzca al estrés (Del Hoyo, 2001). Además, hay que tener en cuenta que los aspectos personales pueden variar en el tiempo en función de factores tales como la edad, las necesidades y expectativas y los estados de salud y fatiga. Obviamente, todos estos factores actúan entre sí e influyen en el estrés experimentado por el trabajador, tanto en el puesto de trabajo como fuera de él.

Por lo tanto, estas variables suponen discrepancias o desajustes entre las demandas del ambiente y los recursos disponibles. De este modo, la importancia que el trabajador le dé a esas discrepancias será definitiva para que incidan en él de una forma más o menos negativa. En este sentido caben destacar dos principales grupos de fenómenos cuyo peso es relevante en estos procesos: 1) características inherentes al trabajador como autoeficacia, locus de control, valores, personalidad resistente, personalidad tipo A, las estrategias de afrontamiento), y 2) la experiencia subjetiva, especialmente lo que tiene que ver con la entidad de la discrepancia entre las demandas del entorno y de los recursos de que dispone para hacerles frente.

Las características de las personas no tienen un carácter estático con respecto a las demandas del entorno, sino que las expectativas, deseos y necesidades de la persona tienen un efecto activo en la relación con el entorno laboral y, en último término, en la generación del estrés. Así, el modo de actuación de estas características en la generación del estrés proviene de la descompensación, desequilibrio o incongruencia entre lo que exteriormente es demandado y lo que los individuos necesitan, desean, o son capaces de hacer. La interrelación que se da entre las demandas del trabajo y las características de la persona no es de carácter automático ni es una comparación de carácter objetivo, sino que está mediatizada por la particular percepción que el individuo tiene de ella y, por lo tanto, es influenciada por aspectos subjetivos (Del Hoyo, 2001); es más, en la generación del estrés, la significación e incluso la existencia de una posible situación de desajuste o desequilibrio proviene de la apreciación que el sujeto haga de esa situación. Por ello, en la generación del estrés resulta muy importante comprender cómo el sujeto experimenta sus necesidades, deseos y expectativas en relación con lo que el entorno ofrece o demanda (Morán, 2006).

Entre los rasgos de personalidad que se ha demostrado que tienen una influencia importante en el proceso y generación de estrés estarían los patrones de conducta (formas de comportamiento que pueden incidir directamente en la aparición o no de síntomas), mientras que en cuanto a los estilos cognitivos hay que decir que éstos intervienen de forma evidente en los procesos de evaluación primaria y secundaria, pero también indudablemente en la generación de estrategias de afrontamiento, en su puesta en práctica y en la reevaluación del proceso (Morán, 2006). Una de las características cognitivas principales que influyen en las respuestas a los estresores, es el "locus de control" (Pérez, 1985), que hace referencia a las explicaciones que una persona se da a sí misma sobre las 
cosas que le suceden. En un extremo estarían las personas con un "locus de control externo", que son las que atribuyen los sucesos que les acontecen a circunstancias externas, que dependen de la suerte o el destino. En el otro extremo, estarían las personas con un "locus de control interno", que son las que atribuyen los sucesos que les acontecen a circunstancias internas. Estos perciben que tienen control de la situación, lo que influye en las expectativas de afrontamiento, experimentando menos amenaza ante los estresores que los externos y respondiendo más adecuadamente, ya que buscan información y se enfrentan al problema. En el ámbito ocupacional se ha señalado que ambos externos presentan más experiencias de estar quemado, de insatisfacción y de estrés laboral. No obstante, en la medida en que un individuo juzga que el mismo tiene el control en una situación, será menos probable que perciba la situación como amenazante y que manifiesten patrones de reacción adversos (Llaneza, 2009).

Por último, y al margen de los factores precipitantes y de vulnerabilidad que pueden describir la situación de estés, debemos señalar que también existen otros factores muy importantes que configuran una mayor resistencia del trabajador ante este tipo de problemas, entre los que destacan el apoyo social, las adecuadas estrategias de afrontamiento y la personalidad resistente (Morán, 2006), aspectos que sin duda también deberán ser evaluados por el profesional que atienda a este tipo de personas.

\subsection{Burnout}

El llamado síndrome de Burnout, habitualmente traducido como "síndrome del quemado" o "síndrome de quemarse en el trabajo", podría entenderse como una respuesta al estrés laboral crónico que incluye sentimientos displacenteros y actitudes negativas hacia las personas con las que se trabaja y hacia el trabajo en sí mismo (Gil-Monte \& Peiró, 1997; Ramos \& Buendía, 2001). Posiblemente la definición más aceptada internacionalmente sobre el Burnout sea la propuesta por Maslach y Jackson en 1986, según la cual se trata de un síndrome de agotamiento emocional, despersonalización y baja realización personal, que puede ocurrir entre individuos que trabajan con personas. Este fenómeno es considerado como un síndrome porque los síntomas que origina parecen agruparse de una manera sistemática en las personas que lo sufren (Ramos, 1999), apareciendo habitualmente cansancio o agotamiento emocional, despersonalización y sentimiento de falta de realización profesional o disminución de la competencia profesional percibida. El burnout se suele definir como una situación de desgaste profesional que sufren los trabajadores que realizan tareas para personas (sanidad, enseñanza, administración pública, policía, cuerpos de emergencias, servicios sociales) debido a unas condiciones de trabajo con elevadas demandas sociales; se trata por tanto de un tipo de respuesta prolongada ante estresores emocionales e interpersonales crónicos en el lugar de trabajo (Luna, 2004; Morán, 2006; Sauter et al., 1998).

Tal y como hemos señalado, este síndrome se caracteriza por el agotamiento emocional (ej., disminución y pérdida de energía, fatiga), la despersonalización (ej., desarrollo de actitudes negativas, de insensibilidad y respuestas frías e impersonales hacia los receptores del servicio prestado) y la baja realización personal ( $p$. ej., tendencia a evaluar el propio trabajo de forma negativa) (Maslach, 1976; Maslach \& Jackson, 1981, 1986). Pero además de estas características, han de producirse dos aspectos asociados al desempeño laboral: 1) la existencia de una relación interpersonal directa entre el trabajador y los usuarios cargada de emoción (principalmente negativa) y 2) el intento en algún momento de superar las limitaciones de la situación mediante la inversión emocional del trabajador (valor, constancia, nobleza). El fracaso de este intento de afrontamiento condicionará el cuadro clínico desarrollado a posteriori.

Respecto a las consecuencias asociadas a este síndrome, debemos señalar que el agotamiento es la manifestación primaria del síndrome y su dimensión central (Morán, 2006; Ramos \& Buendía, 2001; Peiró, 2005). Cuando las personas que sufren burnout describen su propia experiencia relatan fundamentalmente esta sensación de agotamiento, cansancio o sentimiento de estar exhausto (Maslach \& Jackson, 1986). Se trata de la dimensión más significativa del propio síndrome, por lo que algunos investigadores han llegado a argumentar que las otras dimensiones son innecesarias (Pines \& Aronson, 1988). Pero 
esta fuerte identificación del burnout con el agotamiento no es un criterio suficiente para caracterizarlo; el agotamiento es el componente individual del Burnout, pero no puede quedarse fuera del contexto porque, coincidiendo con Ramos(1999) se perdería la perspectiva del fenómeno.

Por otra parte, la despersonalización es un intento de distanciarse del receptor de los servicios ignorando activamente las cualidades que les hacen personas únicas; siendo sus demandas más fáciles de gestionar cuando se les considera objetos impersonales del trabajo (Morán, 2006). Esta dimensión fue descrita por Maslach y Jackson (1986) como la respuesta impersonal y fría, en ocasiones insolente y desconsiderada, hacia los receptores de los servicios o cuidados del profesional. El distanciamiento es una reacción tan inmediata al agotamiento que en toda la investigación sobre el burnout aparece constantemente la relación entre agotamiento y despersonalización es una gran variedad de ámbitos organizativos y profesionales (Maslach et al., 2001).

Respecto a la falta de realización personal, se debe señalar que la ineficacia se relaciona con las otras dos dimensiones del burnout, pero ésta es más compleja. En algunos casos parece depender, hasta cierto punto, del agotamiento, de la despersonalización o de una combinación de ambos (Lee \& Ashforth, 1996).

Por último, debemos señalar que al margen de los indicadores hasta ahora expuestos, resulta habitual que los trabajadores expuestos a este tipo de riesgo psicosocial lleguen a presentar distinta sintomatología clínica que incluye distintos síntomas psicosomáticos (ej., cefaleas, algias corporales, musculares y osteoarticulares, cambios significativos de peso (+/-), úlceras, desordenes gastrointestinales), emocionales (ej., irritabilidad, ansiedad generalizada y focalizada al trabajo, depresión, frustración, sentimientos de inutilidad, aburrimiento) y conductuales (ej., absentismo laboral, abuso de drogas (café, tabaco, alcohol, fármacos) relaciones frías y distantes, conductas de riesgo, llanto inespecífico (Gil-Monte y Peiró, 1997).

\subsection{Mobbing}

El acoso psicológico en el lugar de trabajo, fenómeno también conocido con el anglicismo mobbing, ha sido descrito como una forma de violencia psicológica que tiene lugar en el ámbito laboral y es susceptible de ser manifiesta a través de muy diversas conductas cometidas sobre una persona de forma sistemática (al menos una vez a la semana) y durante un tiempo prolongado (al menos durante seis meses) (Leymann, 1996; Martín-Daza et al., 1998).

A nivel internacional, el mobbing o acoso psicológico en el lugar de trabajo se ha descrito como el conjunto de comportamientos de hostigamiento interpersonal que tienen lugar en el ámbito laboral y que pueden de ser expresados de forma muy variada a través de distintos tipos de aparentes conflictos (Piñuel, 2008). Estos fenómenos se verían facilitados por el hecho de que en las organizaciones de trabajo suelen estar contempladas únicamente las relaciones de carácter laboral y no así las de tipo interpersonal (González-Trijueque, 2007; Pérez-Bilbao et al., 2001). De hecho, el mobbing está considerado como una forma de estrés laboral que presenta la particularidad de no producirse exclusivamente por causas directamente relacionadas con el desempeño del trabajo o con su organización, sino que tiene su origen en las relaciones interpersonales que se establecen dentro de cualquier organización entre los distintos individuos que la conforman (Piñuel, 2001).

Las definiciones sobre mobbing son variadas, pero en esencia definen características muy similares. Así por ejemplo, en España, el acoso laboral ha sido definido desde el Instituto Nacional de Seguridad e Higiene en el Trabajo (INSHT) como "una situación en la que una persona ejerce una violencia psicológica extrema, de forma sistemática y recurrente (como media una vez por semana) y durante un tiempo prolongado (como media unos seis meses) sobre otra persona o personas en el lugar de trabajo con la finalidad de destruir las redes de comunicación de la víctima o víctimas, destruir su reputación, perturbar el ejercicio de sus labores y lograr que finalmente esa persona o personas acaben abandonando su lugar de trabajo" (Martín-Daza et al., 1998). 
Partiendo de esta definición, similar a otras definiciones, se debe destacar que el acoso laboral no debe ser confundido con los habituales conflictos interpersonales que pueden producirse en las organizaciones de trabajo, ni con situaciones de estrés laboral propiamente dichas, ni con otros riesgos psicosociales como por ejemplo, Burnout (González-Trijueque, 2007).

Los datos alcanzados hasta la fecha han resultado muy heterogéneos debido a las dificultades en la definición conceptual del constructo mobbing, los diferentes instrumentos de medida utilizados y el diverso origen sectorial de las muestras de estudio entre otros aspectos (Einarsen et al., 2003; González-Trijueque, 2007). Por ello, se antoja complicado poder ofrecer unas conclusiones absolutas en materia de acoso laboral; situación que, sin embargo, no ha impedido que a partir de las definiciones más operativas sobre el concepto mobbing se hallan desarrollado valiosas investigaciones de referencia (Zapf et al., 2003). No obstante, y pese a ser evidente que el estudio del mobbing necesita de un mayor desarrollo (Einarsen, 2000), las investigaciones desarrolladas se han centrado en intentar conocer la etiología del acoso psicológico laboral y han procurado precisar las distintas variables que intervienen en su dinámica, agrupándose los principales estudios en tres líneas de investigación: 1) sobre las características del acosador y la víctima; 2) sobre las características inherentes a las relaciones interpersonales dentro de las organizaciones de trabajo; y 3 ) sobre los riesgos psicosociales existentes en el entorno laboral.

Al margen de las distintas líneas de investigación existentes, en los que sí hay consenso generalizado es en, considerar el mobbing como un fenómeno de origen claramente multicausal (Einarsen \& Hauge, 2006; Zapf et al., 2003) y en sus negativos efectos, tanto para el trabajador afectado como para su esfera sociofamiliar y la propia organización de trabajo (Einarsen \& Mikkelsen, 2003; González de Rivera \& Rodríguez-Abuín, 2006; González-Trijueque \& Delgado, 2008; Leymann \& Gustafsson, 1996; Matthiesen \& Einarsen, 2001, 2004; Mikkelsen \& Einarsen, 2001, 2002a, 2002b).

\section{MARCO LEGAL INTERNACIONAL Y SITUACIÓN EN URUGUAY}

Las graves consecuencias asociadas a la exposición de los riesgos psicosociales han llevado a diversos organismos internacionales como la Organización Mundial de la Salud (OMS) o la Organización Internacional del Trabajo (OIT), a señalar la importancia del problema y a impulsar grupos de trabajo y estudios que permitan un conocimiento más detallado de la situación y de las estrategias de prevención e intervención más adecuadas. Así, diversos países de la Unión Europea (UE) han comenzado a legislar de modo expreso y específico algunos de los principales riesgos psicosociales. La importancia de estos riesgos es hoy en día innegable y tanto es así que la misma UE los ha considerado como actuación prioritaria en sus estrategias sobre seguridad y salud laboral desde el año 2002, de hecho, la normativa laboral obliga a que todas las organizaciones tengan realizada la evaluación de riesgos psicosociales y la correspondiente planificación de su prevención. Además, es preciso recordar que, más allá de la obligación legal, la intervención sobre los riesgos psicosociales, además de los efectos evidentes en la salud, también puede tener un impacto positivo en el aumento de la calidad y rendimiento de la producción o servicio.

Posiblemente sea el problema del mobbing el que ha generado mayor debate en los últimos años en relación a su regulación legal. Al ser un problema que afecta a la salud y seguridad de los trabajadores, ha sido motivo de regulación legal sobre todo en la UE -en general-; en países como Suecia o Noruega, se ha puesto el énfasis en la necesidad de desarrollar marcos legales de tipo laboral preventivo. Otros países como Alemania, Francia o Bélgica han subrayado que la regulación legal de este fenómeno, se diligencie desde la jurisdicción penal (González-Trijueque et al., 2011). Por otra parte, en España, el abordaje legal del mobbing puede llevarse a cabo por cualquier jurisdicción (penal, civil, social, contencioso-administrativa), si bien es cierto que la tipificación del mismo como delito es aún muy reciente -diciembre de 2010-, situación que ha incidido en que sean las jurisdicciones de carácter laboral, social y contencioso-administrativa las que mayor protagonismo han tenido hasta la fecha, con 
especial relevancia de la Ley de Prevención de Riesgos Laborales tomada del modelo sueco (González-Trijueque et al., 2011).

En cuanto a Latinoamérica, el interés por legislar el mobbing apenas ha trascendido, ya que sólo Colombia (Ley 1.010 del año 2006) y Brasil (Ley 2.120 de la administración municipal de Ubatuba del año 2001; Ley complementaria 12.561 de Río Grande do Sul del año 2006; Ley contra el asedio moral del estado de Sao Paulo del año 2007) cuentan con una legislación específica que regule esta problemática sociolaboral (Oceguera et al., 2009). Además, existen distintas leyes de carácter general que regulan el mobbing en países como Argentina (Ley 7.232 de la provincia de Tucumán del año 2002; Ley 5.349 de la provincia de Jujuy del año 2003; Ley 13.168 de la provincia de Buenos Aires del año 2004; Ley 1.225 de la ciudad de Buenos Aires del año 2004; Ley 12.434 de violencia laboral argentina del año 2005; Ley 9.671 de la provincia de Entre Ríos del año 2006) y Venezuela (Ley orgánica de prevención, condiciones y medio ambiente de trabajo del año 2005) (Oceguera et al., 2009). Tal y como puede observarse, únicamente Colombia y Venezuela cuentan con leyes de carácter nacional en toda Latinoamérica que regulen el fenómeno del mobbing, siendo la ley colombiana de carácter específico y la venezolana de carácter más general (González-Trijueque et al., 2011).

Respecto al caso concreto de Uruguay, se puede señalar que no existe ninguna ley específica en relación a los riesgos psicosociales en el lugar de trabajo y de hecho, el Banco de Seguro del Estado -entidad de seguros de carácter estatal que atiende los asuntos relacionados con los perjuicios y problemas generados por y en situaciones laborales- no los reconoce como enfermedades profesionales, por lo que quedan fuera del marco legal uruguayo, al menos de forma directa. Resulta llamativo en este sentido que no existan herramientas legales específicas para luchar contra el problema del acoso laboral en Uruguay, sobre todo cuando los pocos estudios existentes sugieren datos muy alarmantes. Si bien es cierto que, en Uruguay no se han desarrollado hasta la fecha investigaciones empíricas relevantes respecto al mobbing en cuanto a su prevalencia o a sus consecuencias, podemos señalar que, siguiendo a Tapia (2010), las cifras de prevalencia orientativas -obtenidas a través de encuestas- han llegado a cifrar en un $59 \%$ los sospechados de acoso laboral dentro de la población activa uruguaya según datos facilitados por la Asociación de Trabajadores de la Seguridad Social a una de las firmantes del presente artículo, Dña. Silvana Giachero, considerándose además que, una gran mayoría de los casos tienen lugar en el ámbito público. Resulta evidente que en Uruguay no se dispone de datos más precisos sobre la materia, sin embargo, no se debe obviar que los primeros indicios sugieren la presencia de unas elevadas cifras de prevalencia que, sin embargo, no han derivado en el desarrollo de un cuerpo normativo específico, si bien en la actualidad se discute a nivel parlamentario si se establece una ley que regule los vínculos laborales, previniendo y sancionando el maltrato psicológico, el daño moral y la discriminación en el lugar de trabajo.

Esta aparente situación de indefensión, no ha impedido que los trabajadores acosados buscaran amparo en la normativa existente. Con respecto a la legislación uruguaya susceptible de proteger a los trabajadores contra la violencia psicológica y la discriminación laboral, debemos señalar que si bien no existe una legislación específica, sí hay distintas normas que pueden amparar a las víctimas de forma indirecta. Destaca sobremanera el marco normativo más importante, la Constitución de la República Oriental del Uruguay de 1967 (con las correspondientes modificaciones realizadas en 1989, 1994, 1996 y 2004), ya que en dicho texto se recoge (en los artículos $7,8,54,72,332,309,310,311$ y 312) que los derechos de los habitantes de la República han de ser protegidos en igualdad de condiciones (Goyena, 2008). Posteriormente, se han desarrollado distintas leyes que, indirectamente pueden guardar relación con la problemática descrita, así por ejemplo: mediante la ley 12.030 se aprueban convenios adoptados por la OIT y se establece un régimen de sanciones para los infractores. La ley 16.045 prohíbe toda discriminación que viole el principio de igualdad de trato y oportunidades, para ambos sexos, en cualquier sector o ramo de actividad laboral o la ley 18.561 sobre la prevención y sanción del acoso sexual en el ámbito laboral y en las 
relaciones docente-alumno. Por último, no se debe olvidar la relevancia del Código Civil de Uruguay (y su correspondiente norma legal procesal, el Código General del proceso, Ley 15.982) que regula las materias relacionadas con los daños y perjuicios que puedan sufrir las personas.

Fue a partir del año 2005 cuando se comenzó a percibir un cambio en la sociedad uruguaya respecto a la sensibilización hacia este tipo de problemas socio-laborales; así, tanto en el ámbito privado como en el público, se ha detectado un incremento significativo en las denuncias por acoso laboral y otras conductas discriminatorias en los últimos años. Además, recientemente se ha comenzado a abordar el problema del mobbing en los medios de comunicación uruguayos, han surgido numerosas webs en internet relacionadas con la materia, los sindicatos imparten información y realizan actos de divulgación; se comienzan a realizar evaluaciones psicotécnicas sobre estrés laboral en las áreas de salud ocupacional y se crean asociaciones de afectados orientadas a luchar contra este severo perjuicio socio-laboral. Todo ello hace pensar en un significativo avance social en la lucha contra este tipo de riesgos y sus nocivas consecuencias.

Dentro de esta realidad social (y las limitaciones normativas existentes), los trabajadores en Uruguay han comenzado a emprender acciones legales civiles, habiéndose logrado alguna que otra sentencia favorable, situación de gran relevancia a la hora de configurar antecedentes y jurisprudencia al respecto. Hasta la fecha, en Uruguay, los casos de acoso moral laboral han sido tratados y juzgados como supuestos de despido indirecto, teniendo que abonar el empleador al trabajador la indemnización correspondiente. Sin embargo, debido al aumento de casos en los últimos años y a que se ha producido un cambio en la modalidad de hostigamiento hacia un acoso más explícito, con un aumento significativo en las licencias de baja por enfermedad psiquiátrica de los trabajadores (principalmente por ansiedad, depresión e incluso suicidios), el Ministerio de Trabajo y Seguridad Social (MTSS) ha abierto una oficina específica para atender estos casos, entrenando y capacitando a abogados e inspectores sobre la materia. De hecho, en 2011 se ha firmado un convenio con el área de psicología del trabajo y las organizaciones de la facultad de Psicología de la Universidad de la República, con el fin de capacitar más profesionales e inspectores.

Actualmente, se estima que las denuncias que se reciben en el MTSS sobre riesgos psicosociales laborales son de, un $70 \%$ en el ámbito privado y un $30 \%$ en la administración pública. Pero debemos tener en cuenta que en Uruguay sólo se actúa realizando inspecciones cuando la denuncia es de un trabajador del ámbito privado, mientras que si la denuncia es de un trabajador de la administración pública, el único recurso de que dispone es solicitar una investigación administrativa, la que monitorea el MTSS, para que no se pierda y se realice en tiempo y forma establecidos. De lo que surge que el funcionario público, se encuentra más desprotegido legalmente. Ello implica que los casos más graves (y la mayor cantidad de ellos) se estén dando en el ámbito público.

\section{LOS APORTES DE LA PSICOLOGÍA APLICADA}

Resulta evidente que los riesgos psicosociales en el lugar de trabajo configuran una problemática socio-laboral de gran interés a nivel internacional, para las que se deben adoptar medidas para paliar en la medida de lo posible sus negativas consecuencias. En este sentido, la prevención de riesgos laborales en el marco de su disciplina como la psicosociología aplicada, se antoja fundamental.

La forma de abordar la prevención de riesgos laborales, en el área de ergonomía y la psicosociología aplicada, se puede entender de varias formas, pero la mayor parte de las posturas se agrupan en dos grandes enfoques. Por un lado la orientación proactiva y por otro la orientación reactiva; entre los partidarios de ambas posturas se ha creado un interesante debate que, en esencia, afecta a la eficiencia de métodos y a la distribución de los recursos existentes (Llaneza, 2009). De entrada es necesario clarificar las posturas, así pues describiremos ambos enfoques. Entendemos como enfoque proactivo, aquel destinado a evitar la aparición de elementos dañinos en el entorno laboral, o preparar personas y organizaciones para minimizar su impacto, en caso de ser inevitables. Este es un 
enfoque coherente con el ideario clásico de la prevención. Por otra parte, el enfoque reactivo es, paradójicamente, partidario de aceptar de entrada la inevitabilidad de la aparición de un elemento dañino, dando como solución viable reaccionar ante él, proveyendo al individuo y a la organización de los mecanismos de compensación más eficaces posibles. Muchos autores defienden que el único enfoque preventivo real es el proactivo, y que la orientación reactiva no es siquiera prevención, en un sentido estricto.

Resulta clarificador -llegados a este punto- describir los distintos tipos de riesgos que abordan los dos enfoques. Los defensores del enfoque proactivo, se centran principalmente en los aspectos ergonómicos y en aquellos elementos que pueden ser medidos con relativa facilidad (presión de tiempo, fatiga, sobrecarga laboral, etc.) y, con buen criterio, establecen que una correcta evaluación previa puede minimizar e incluso eliminar la aparición de daño, como consecuencia de la activación de uno de estos riesgos. Por otra parte, los defensores del enfoque reactivo están más dirigidos a los llamados nuevos riesgos, los que contempla la psicosociología relacional (PSR): el mobbing, el Burnout y los diferentes tipos de discriminaciones; $y$ consideran que no es posible, al menos de momento, establecer ninguna medida de prevención útil frente a estos riesgos que permita un enfoque proactivo, y defienden que una eficiente red de detección precoz e intervención inmediata es la mejor medida posible.

Este principio de separación entre ambas posturas, es coherente con otra línea de pensamiento actual que trata de separar la psicosociología aplicada de la ergonomía, como áreas especificas y separadas de la prevención laboral; siendo los diferentes enfoques sobre los supuestos de riesgos, una base práctica para la escisión del área de prevención actual (ergonomía y psicosociología Aplicada) en dos entidades independientes, con metodologías y enfoques estratégicos específicos y diferenciados. No obstante, es necesario apuntar que ambas partes comprenden que, los enfoques reactivo y proactivo, son los extremos de un continuo en el cual pueden convivir ambas filosofías, y reconocen la necesidad urgente de mejorar los métodos de medida e incrementar las evaluaciones psicosociales; asimismo convienen como objetivo deseable llegar a un enfoque mayoritariamente proactivo en un futuro indeterminado. Por lo tanto, las posiciones se establecen alrededor de una aproximación teórica a porcentaje de medidas y recursos (económicos, técnicos y humanos) que deben adjudicarse a uno u otro enfoque.

Ciñéndonos a lo expuesto anteriormente, es perfectamente plausible enfocar la prevención de riesgos de tipo ergonómico de forma proactiva; y asimismo es lógico asumir el enfoque reactivo para los riesgos psicosociales (especialmente los de tipo relacional); por lo que, quizás la medida más adecuada sea desarrollar textos normativos de tipo prevencionista siguiendo el modelo sueco, independientemente de que por otra parte se penalice la conducta del acosador.

\section{CONCLUSIONES}

Reconociendo la limitación real de que, es un hecho, que aún no podemos prevenir con garantías los riesgos referidos a asuntos psicosociales como el mobbing, el burnout o la discriminación, la conclusión principal es que la única opción responsable es crear y disponer de equipos de respuesta precoz, con la mayor eficiencia posible, en los lugares de trabajo; mientras se destinan recursos (minoritarios) a la investigación y prueba de métodos proactivos. Sobre el cómo crear y ubicar estos equipos de intervención precoz, otros profesionales ya han apuntado posibles soluciones. La primera propuesta que realizamos es la de incluir un psicólogo, sociólogo o psicosociólogo en las organizaciones de trabajo; figura que tendría un peso y una función equivalente a la del médico de empresa. La segunda estriba en que, los servicios de prevención ajenos y propios, así como, las instituciones que atienden los accidentes de trabajo y enfermedades profesionales, dispongan de un equipo de atención rápida a demandas psicosociales; de formato y funcionamiento similar al de los equipos de emergencia sanitaria. Ambas propuestas tienen inconvenientes; en el caso del "psicosociólogo de empresa" aparecerían problemas de dinámica interpersonal debido a que, como es sabido, las relaciones entre consultor-terapeuta y paciente-cliente se basan en la confianza y en la seguridad de que se mantendrá el compromiso de privacidad. Resulta difícil creer que un empleado pueda confiar en un profesional pagado por su empresa, especialmente si sus 
motivos de queja son contra la misma empresa. Respecto a la segunda solución, los "equipos de emergencia", si bien parece defendible desde la teoría, cuenta con gran cantidad de detractores, especialmente desde las mutuas o instituciones de asistencia de problemas laborales y algunos servicios de prevención ajenos, ya que complica aún más la carencia de profesionales preparados para formar estos equipos.

Para concluir, podemos señalar que lo que resulta evidente es que en Uruguay, al igual que en otros muchos países, no se está abordando satisfactoriamente este tipo de problemas, que por otra parte se sabe que ocasionan alto nivel de sufrimiento a quienes lo padecen, así como, otro tipo de problemas de funcionamiento a las organizaciones donde se producen e incluso, costes de tipo económico a los estados que tienen que hacerse cargo, finalmente, de muchas de las consecuencias asociadas. Es por ello, que parece fundamental continuar investigando y difundiendo sobre este tipo de problemática, para formar profesionales y desarrollar los imprescindibles marcos normativos que amparen a quienes sufren estos "nuevos riesgos emergentes".

\section{REFERENCIAS}

Almodóvar, M. A., Berjón, M. A., Cuenca, M. R., Fraile, A., García de Castro, M., Del Hoyo, M. A., Martín-Daza, F., Nogareda, C., Nogareda, S., Oncins, M., Urrutia, M. \& Zubizarreta, I. (2003). Psicosociología del trabajo. Madrid: Instituto Nacional de Seguridad e Higiene en el Trabajo.

Buendía, J. \& Ramos, F. (2001). Empleo, estrés y salud. Madrid: Pirámide.

Del Hoyo, M. A. (2001). Estrés laboral. Madrid: Instituto Nacional de Seguridad e Higiene en el Trabajo.

Einarsen, S. (2000). Harassment and bullying at work: A review of the Scandinavian approach. Aggression and Violent Behaviour, 5(4), 379-401.

Einarsen, S. \& Hauge, L. J. (2006). Antecedentes y consecuencias del acoso psicológico en el trabajo: una revisión de la literatura. Revista del Trabajo y de las Organizaciones, 22(3), 251-274.

Einarsen, S., Hoel, H., Zapf, D. \& Cooper, C. L. (2003). The concept of bullying at work: The European tradition. En S. Einarsen, H. Hoel, D. Zapf y C. L. Cooper (Eds.), Bullying and emotional abuse in the workplace. International perspectives in research and practice. London: Taylor and Francis.

Einarsen, S. \& Mikkelsen, E. G. (2003). Individual effects of exposure to bullying at work. En S. Einarsen, H. Hoel, D. Zapf y C. L. Cooper (Eds.), Bullying and emotional abuse in the workplace. International perspectives in research and practice. London: Taylor and Francis.
Gil-Monte, P. \& Peiró, J. M. (1997). Desgaste psíquico en el trabajo. El síndrome de quemarse. Madrid: Síntesis.

González de Rivera, J. L. \& Rodríguez-Abuín, M. (2006). Acoso psicológico en el trabajo y psicopatología: un estudio con el LIPT-60 y el SCL-90-R. Revista del Trabajo y de las Organizaciones, 22(3),397-412.

Gonzalez de Rivera, J. L. \& Rodríguez-Abuin, M.,J.(2005). Cuestionario de estrategias de acoso en el trabajo. EI LIPT-60. EOS: Madrid.

González-Trijueque, D. (2007). El acoso psicológico en el lugar de trabajo: una aproximación desde la psicología forense. Psicopatología Clínica Legal y Forense, 7, 41-62.

González-Trijueque, D. \& Delgado, S. (2008). Acoso psicológico en el lugar de trabajo, burnout y psicopatología: Un estudio piloto con el BSI y el MBI. Boletín de Psicología, 94, 47-56.

González-Trijueque, D. \& Graña, J., L. (2010). Profiles of social skills, coping strategies and perceived social support in mobbed workers. Psicopatología Clínica Legal y Forense, 10, 7-22.

González-Trijueque, D., Tejero, R. \& Delgado, S. (2011). El mobbing desde la perspectiva de la psicología jurídica. En G. A. Hernández-Medina (Dir.), Psicología Jurídica Iberoamericana. Bogotá: El Manual Moderno.

Goyena, P. V. (2008). La Constitución de la República Oriental de Uruguay. Montevideo: Bibliolife.

Lee, R. T. \& Ashforth, B. E. (1996). Ameta-analytic examination of the correlates of the three dimensions of job burnout. Journal of Applied Psychology, 81(2), 123-133.

Leymann, H. (1996). The content and development of mobbing at work. European Journal of Work and Organizational Psychology, 5(2), 165-184.

Leymann, H. \& Gustafsson, A. (1996). Mobbing at work and the development of post-traumatic stress disorder. European Journal of Work and Organizational Psychology, 5(2), 251-275.

Llaneza, F. J. (2005). La ergonomía forense. Pruebas periciales en prevención de riesgos laborales. Valladolid: Lex Nova.

Llaneza, F. J. (2009). Ergonomía y psicosociología aplicada. Manual para la formación del especialista. Valladolid: Lex Nova.

Guélaud, F., Beauchesne, M.N., Gautrat, J. \& Roustang,G. (1978). Método LEST (Laboratorio Aix-en-Provence). En J.J. Castillo \& C. Prieto (1990). Condiciones de trabajo. Un enfoque renovador de la sociología del trabajo. Madrid: Centro de Investigaciones Sociológicas.

Luna, A. (2004). Psicopatología laboral. En J. A. Gisbert (Dir.), Medicina legal y toxicología. Barcelona: Masson.

Martín-Daza, F., Pérez-Bilbao, J. \& López, A. (1998). NTP 476. El hostigamiento psicológico en el trabajo: mobbing. Madrid: Instituto Nacional de Seguridad e Higiene en el Trabajo.

Maslach, C. (1976). Burned-out. Human Behavior, 5, 16-22.

Maslach, C. \& Jackson, S. E. (1981). The measurement of experienced burnout. Journal of Occupational Behavior, 2, 99-133.

Maslach, C. \& Jackson, S. E. (1986). Maslach Burnout Inventory. Manual Research Edition. Palo Alto, CA: Consulting Psychologist Press.

Maslach, C., Schaufeli, W. B. \& Leiter, M. P. (2001). Job Burnout. Annual review of psychology, 52, 397-422. 
Matthiesen, S. B. \& Einarsen, S. (2001). MMPI-2 configurations among victims of bullying at work. European Journal of Work and Organizational Psychology, 10(4), 467-484.

Matthiesen, S. B. \& Einarsen, S. (2004). Psychiatric distress and symptoms of PTSD among victims of bullying at work. British Journal of Guidance and Counselling, 32(3), 335-356.

Mikkelsen, E., G. \& Einarsen, S. (2001). Bullying in Danish work-life: Prevalence and health correlates. European Journal of Work and Organizational Psychology, 10(4), 393-413.

Mikkelsen, E. G. \& Einarsen, S. (2002a). Basic assumptions and symptoms of post-traumatic stress among victims of bullying at work. European Journal of work and Organizational Psychology, 11(1), 87-111.

Mikkelsen, E. G. \& Einarsen, S. (2002b). Relationships between exposure to bullying at work and psychological and psychosomatic health complains: The role of state negative affectivity and generalized self-efficacy. Scandinavian Journal of Psychology, 43, 397-405.

Moncada, S., Llorens, C. \& Kristensen, T. S. (2002). Método ISTAS 21. Manual para la evaluación de riesgos psicosociales en el trabajo. Madrid: Instituto Sindical de Trabajo, Ambiente y Salud.

Moncada, S., Llorens, C. \& Kristensen, T. S.(2005). ISTAS21 COPSOQ: Versión en lengua castellana del cuestionario psicosocial de Copenhague, Archivos de Prevención de Riesgos Laborales 8, no.1, 18-29

Morán, C. (2006). Estrés, burnout y mobbing. Recursos y estrategias de afrontamiento. Madrid: Amaru.

Oceguera, A., Aldrete, G. \& Ruiz-Moreno, A. G. (2009). Estudio comparado de la legislación del mobbing en Latinoamérica. Política y Sociedad, 8, 83-94.

Peiró, J. M. (1999). Desencadenantes del estrés laboral. Madrid: Pirámide.
Pérez-Bilbao, J., Nogareda, C., Martín-Daza, F. \& Sancho, T. (2001). Mobbing, violencia física y acoso sexual. Madrid: Instituto Nacional de Seguridad e Higiene en el Trabajo.

Perez, J. (1985) El modelo de J.B. Rotter. En J. Bermudez (Dir.). Psicología de la personalidad. Madrid: UNED

Pines, A. \& Aronson, E. (1988). Career burnout. Causes and cures. New York: The Free Press.

Piñuel, I. (2001). Mobbing. Cómo sobrevivir al acoso psicológico en el trabajo. Santander: Sal Térrae.

Ramos, F. (1999). El síndrome de burnout. Madrid: Universidad Nacional de Educación a Distancia (UNED).

Ramos, F. \& Buendía, J. (2001). El síndrome de burnout: concepto, evaluación y tratamiento. En J. Buendía y F. Ramos (Coords.), Empleo, estrés y salud. Madrid: Pirámide.

Sauter, S. L., Murphy, L. R., Hurrell, J. J., \& Levi, L. (1998). Factores psicosociales y de organización. En J. M. Stellman (Dir.), Enciclopedia de Salud y Seguridad en el Trabajo. Ginebra: OIT.

Tapia, C. (2010, Julio 18). Acoso que se vuelve rutina. El País. Extraído el 2 de Septiembre de 2010 en http:// www.elpais.com.uy/suplemento/ds/Acoso-que-sevuelve-rutina/sds_502268_100718.html

Velázquez, M. (2005). Mobbing. Violencia física y estrés en el trabajo. Aspectos jurídicos de los riesgos psicosociales. Barcelona: Gestión 2000.

Vida, J. (2003). Manual para la formación en prevención de riesgos laborales. Valladolid: Lex Nova.

Zapf, D., Einarsen, S., Hoel, H. \& Vartia, M. (2003). Empirical findings on bullying in the workplace. En S. Einarsen, H. Hoel, D. Zapf y C. L. Cooper (Eds.), Bullying and emotional abuse in the workplace. International perspectives in research and practice. London: Taylor and Francis.

Para citar este artículo:

Trijueque-Gonzalez, D., Giachero, S. \& Delgado, S. (2012). Riesgos psicosociales en el lugar de trabajo: aproximación teórica y marco legal en Uruguay. Ciencias Psicológicas VI (1): 75-87. 